\title{
Molécula de Injúria Renal 1 (KIM-1) no diagnóstico da lesão renal aguda
}

\author{
Taíse Winter Forest, Dirleise Colle*
}

Departamento de Análises Clínicas, Centro de Ciências da Saúde, Universidade Federal de Santa Catarina, Florianópolis, SC, Brasil

Histórico do Artigo

Submetido em:

09/10/2018

Aceito em:

$28 / 11 / 2018$

Palavras-chave:

Lesão renal aguda;

Creatinina sérica,

Molécula de injúria

renal 1 (KIM-1);

Performance clínica.

\begin{abstract}
RESUMO
A lesão renal aguda (LRA) é uma complicação de saúde caracterizada por uma diminuição na taxa de filtração glomerular em um curto período de tempo. Atualmente, o diagnóstico de LRA é baseado, principalmente, na dosagem da creatinina sérica, cuja principal limitação é o diagnóstico tardio. Tendo em vista isso, novos biomarcadores têm sido estudados para o diagnóstico da LRA e vêm apresentando resultados promissores, como é o caso da molécula de injúria renal 1 (KIM-1). O objetivo principal deste estudo foi realizar a revisão crítica atual da literatura sobre o papel da KIM-1 no diagnóstico precoce da LRA. Foi realizada uma busca nas bases de dados Medline e LILACS em publicações dos últimos dez anos. Foram considerados elegíveis estudos em inglês ou em português, nos quais KIM-1 foi dosada na urina como critério diagnóstico de LRA. Foram selecionados e analisados 17 estudos. A concentração urinária de KIM-1 aumentou em um curto período de tempo em pacientes com LRA associada a diversas condições clínicas primárias, apresentado, no geral, boa performance clínica com áreas sob a curva de características operacionais do receptor (AUC-ROC) de 0,57 a 1,0. Os dados avaliados nesta revisão indicam que a KIM-1 urinária apresenta potencial como biomarcador precoce de LRA. Entretanto, estudos futuros são necessários para validar a performance clínica desse biomarcador e, além disso, como não existe um marcador urinário ideal para diagnóstico precoce de LRA, estudos que avaliam a associação de diferentes biomarcadores são importantes no intuito de melhorar a performance diagnóstica dos mesmos.
\end{abstract}

Kidney Injury Molecule 1 (KIM-1) in the Diagnosis of Acute Kidney Injury
Keywords:

Acute kidney injury; Serum creatinine; Kidney injury molecule 1 (KIM-1); Clinical performance.

\begin{abstract}
Acute kidney injury (AKI) is a common health complication, characterized by a rapid decline in the glomerular filtration rate. Currently, the standard diagnostic tool for AKI detection is the monitoring of serum creatinine concentration; however, the mainly limitation of serum creatinine is the late in AKI diagnosis. Therefore, over the last few years several new AKI biomarkers have been studied to improve early detection of AKI, such as the kidney injury molecule 1 (KIM-1). We performed a critical review of the current literature to evaluate potential role of KIM-1 as an early biomarker of AKI. The research was conducted in the Medline and LILACS databases in publications from the last ten years. Primary studies in English or Portuguese were considered eligible, in which KIM-1 was measured in urine samples for the purpose of early diagnostic of AKI in patients. Seventeen articles were selected and analyzed. KIM-1 increased over a short period of time in AKI patients associated with several primary clinical conditions. In addition, KIM-1 presented a good clinical performance with area under the receiver operating characteristics curve (AUC-ROC) from 0.57 to 1.0. The data indicate that KIM-1 is a potential novel urinary biomarker for the early detection of AKI. However, future studies are required to validate its clinical utility. Since no urinary biomarker can meet all the requirements for an ideal biomarker, studies evaluating the combination of different biomarkers are important in order to improve their diagnostic performance.
\end{abstract}

\section{Introdução}

A lesão renal aguda (LRA) é caracterizada por uma redução repentina na taxa de filtração glomerular (TFG) num intervalo de tempo de algumas horas ou dias, gerando a incapacidade dos rins em realizar sua função de excreção (1). Atualmente, a LRA é

\footnotetext{
*Autor correspondente: dirleise.colle@ufsc.br (Colle D.)
} 
definida como um aumento da creatinina sérica de $0,3 \mathrm{mg} / \mathrm{dL}$ em 48 horas ou um aumento de 1,5 vezes o valor basal, ou uma redução da diurese para valores inferiores a $0,5 \mathrm{~mL} / \mathrm{kg} / \mathrm{h}$ por 6 horas (2).

A LRA pode ser desencadeada por diversos fatores como isquemia, sepse, medicamentos e agentes nefrotóxicos, podendo ser potencialmente fatal em pacientes hospitalizados e com patologias graves. A LRA está particularmente relacionada ao ambiente hospitalar, visto que é uma das complicações mais comuns em pacientes hospitalizados (3). A LRA é responsável por $1 \%$ de todas as internações hospitalares, complicando 7\% das mesmas e sua incidência aumenta para 30-50\% em pacientes internados em unidade de terapia intensiva (UTI) (4). Além disso, frequentemente, está associada com diversas complicações tanto a curto como a longo prazo. Embora seja comumente revertida (5), a LRA é responsável por altas taxas de mortalidade e morbidade, podendo evoluir para condições mais graves como a doença renal crônica, doença renal em estágio terminal e, finalmente para a necessidade de terapias de substituição renal. (6-8).

Atualmente, o diagnóstico padrão da LRA é realizado através do monitoramento da concentração sérica de creatinina, a qual representa um marcador de função renal e não de lesão renal (9). Em condições normais da função renal, a creatinina é livremente filtrada pelos glomérulos e excretada na urina. Nestas condições, as concentrações de creatinina sérica situam-se, em média, entre $0,4-1,1 \mathrm{mg} / \mathrm{dL}$ em mulheres e $0,6-1,3 \mathrm{mg} / \mathrm{dL}$ em homens.Quando ocorre um comprometimento na função renal, as concentrações séricas de creatinina aumentam. Apesar da sua análise laboratorial ser muito barata e a mesma apresentar boa estabilidade química na rotina clínica, apresenta marcantes limitações. Em primeiro lugar, suas concentrações variam amplamente conforme o sexo, idade, massa muscular, peso corporal, situação nutricional e estado de hidratação. Segundo, as concentrações de creatinina sérica não se alteram até que haja um significativo comprometimento da função renal. Desta forma, a creatinina sérica é considerada um marcador tardio, visto que quando suas concentrações séricas estão elevadas, já houve uma diminuição de mais de $50 \%$ na TFG $(10,11)$. Baseado na creatinina sérica, o diagnóstico da LRA ocorre somente na fase de diminuição da TGF, quando já ocorreu um substancial dano renal. Após queda da TFG, há um atraso de dias para o aumento da creatinina sérica, o que pode atrasar o diagnóstico em até 72 horas, particularmente em casos de lesão renal modesta (12). Esse atraso em se detectar seu aumento no soro, aliado à baixa sensibilidade e especificidade limita o diagnóstico clínico, o que compromete fortemente uma abordagem terapêutica precoce (13).

Devido às limitações da creatinina sérica no diagnóstico da LRA, nas últimas duas décadas, diversos biomarcadores têm sido identificados para detecção precoce de LRA tais como a molécula de injúria renal 1 (KIM-1), lipocalina associada à gelatinase neutrofílica (NGAL), proteínas de ligação de ácidos graxos (FABPs), interleucina 18 (IL-18), N-Acetil- $\beta$-d-glucosaminidase, entre outros (4, 14-16). Muitos desses novos biomarcadores são marcadores de lesão renal que, em condições normais, estão ausentes ou presentes em concentrações muito baixas, o que aumenta a sensibilidade e a especificidade, visto que muitos deles possuem origens específicas, possibilitando um diagnóstico rápido e preciso (17).

Um biomarcador promissor no diagnóstico da LRA é a proteína KIM-1, uma glicoproteína transmembrana do tipo 1, expressa em baixas concentrações nos rins em estado normal, não sendo detectada no tecido renal ou na urina (18). KIM-1 é um marcador de lesão de células epiteliais tubulares (19), cuja concentração apresenta-se elevada quando há lesão renal isquêmica ou tóxica (17). A expressão de KIM-1 é principalmente aumentada em células tubulares proximais, especialmente no segmento 
S3 tanto em humanos quanto em roedores (20), sendo um marcador de proliferação e regeneração celular no túbulo proximal (21). Além disso, KIM-1 parece estar envolvida tanto no processo de dano renal quanto no processo de recuperação após lesão (22). Ainda, alguns estudos sugerem que KIM-1 participa do processo de fagocitose de células apoptóticas após um evento isquêmico renal (23-25).

Diversos estudos clínicos têm mostrado que poucas horas após a ocorrência de lesão renal os níveis urinários de KIM-1 já se encontram significativamente elevados, enquanto que os níveis séricos de creatinina e a sua depuração renal apenas sofrem alterações significativas entre 24 e $48 \mathrm{~h}$ após a lesão (26-28). Por conta disso, nos últimos anos, o papel da KIM-1 como biomarcador no diagnóstico precoce de LRA associada a diversas condições médicas têm sido extensivamente estudado. Entretanto, os resultados desses estudos mostram uma performance clínica variável na detecção precoce de LRA. Baseado nestes aspectos, o objetivo principal deste estudo foi realizar a revisão crítica atual da literatura sobre o papel da KIM-1 como biomarcador no diagnóstico precoce da LRA.

\section{Materiais e métodos}

\subsection{Métodos de busca dos estudos na literatura}

Foi realizado um levantamento bibliográfico, com buscas nas bases de dados Medical Literature Analysis and Retrieval System Online (MEDLINE) via Pubmed e Literatura Latino-Americana e do Caribe em Ciências da Saúde (LILACS) utilizando os seguintes termos $\mathrm{MeSH}$ ou palavras-chave e suas associações: "Acute kidney injury", "KIM-1" e "Kidney Injury Molecule 1".

\subsection{Seleção dos estudos}

A seleção dos estudos foi realizada por dois pesquisadores independentes (T.W.F. e D.C.). Após a realização da busca utilizando as respectivas palavras-chave e suas associações, os títulos de todos os trabalhos encontrados nas bases de dados foram salvos numa lista inicial. A elegibilidade dos estudos foi inicialmente avaliada através da leitura dos seus títulos e resumos. Em seguida, os trabalhos previamente selecionados, foram lidos na íntegra e avaliados de acordo com os seguintes critérios de inclusão: artigos completos disponíveis e publicados no período de julho de 2007 a julho de 2017; estudos publicados nas línguas inglesa e portuguesa; estudos observacionais e de coorte prospectivos e estudos randomizados; estudos realizados com pacientes maiores de 18 anos diagnosticados para LRA segundo os critérios AKIN (Acute Kidney Injury Network) e KDIGO (Kidney Disease Improving Global Outcomes); trabalhos que realizaram a dosagem do biomarcador KIM-1 em amostras de urina; estudos que utilizaram a curva de características operacionais do receptor (receiver operating characteristics, ROC) e calcularam a área sob a curva ROC (AUCROC), a fim de avaliar a acurácia diagnóstica do marcador. Por outro lado, estudos duplicados; estudos publicados fora do período cronológico estipulado; estudos em línguas diferentes daquelas mencionadas acima; revisões sistemáticas ou narrativas da literatura ou cartas ao editor; estudos experimentais em modelos animais ou em cultivo celular; estudos em que a determinação de KIM-1 era usada como critério diagnóstico sem mensuração da acurácia foram excluídos. Ao final do processo, os artigos selecionados pelos pesquisadores foram comparados e, nos casos de discordâncias entre os dois pesquisadores, estas foram resolvidas por consenso. 


\subsection{Extração e análise dos dados}

Os dados dos artigos selecionados foram extraídos e estruturados mediante formulário de coleta no formato de planilhas no programa Microsoft Excel 2013.

A extração e análise dos dados foram baseadas em a) informações gerais: desenho do estudo, origem do estudo, características dos pacientes, critério diagnóstico de LRA, metodologia empregada na mensuração dos níveis do biomarcador; b) momento da coleta estimando o tempo de insulto renal, quando possível, ou considerando a admissão no serviço de saúde, ponto de corte do exame, performance clínica do biomarcador no diagnóstico precoce de LRA: sensibilidade, especificidade e a AUC-ROC. Os resultados foram apresentados por meio de tabelas e também de forma descritiva.

\section{Resultados}

\subsection{Seleção dos estudos}

A pesquisa inicial nas bases de dados resultou em um total de 713 estudos. Após a exclusão dos registros duplicados restaram 498 estudos, cujos títulos e resumos foram analisados. Destes, 423 artigos foram excluídos por serem revisões sobre o tema, experimentos com animais ou cultivo celular, estudos com crianças, tempo cronológico inferior ao estipulado e estudos em línguas diferentes do inglês e português, restando 75 artigos para a leitura completa. Dentre estes, 17 artigos (26, 27, 29-43) foram incluídos no estudo após leitura na íntegra (Figura 1).

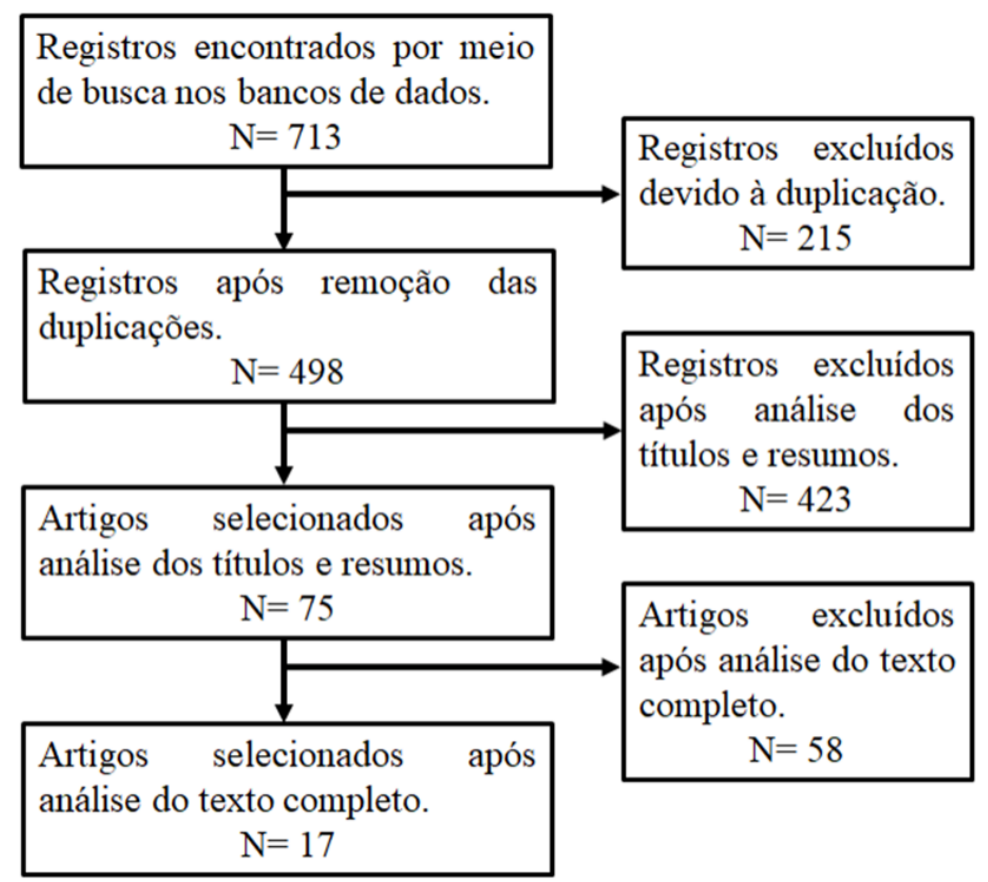

Figura 1 - Fluxograma do processo de seleção dos estudos.

Os bancos de dados consultados foram o Medline através do Pubmed e a plataforma LILACS. Foram obtidos 713 registros. Estudos duplicados ou que não preencheram os critérios de inclusão foram excluídos da presente revisão. 


\subsection{Características dos estudos}

Na tabela 1 estão representadas as características gerais dos estudos selecionados para avaliação do papel da KIM-1 no diagnóstico de LRA. Foram selecionados 17 artigos, sendo na sua maioria estudos de coorte prospectivo ou do tipo observacional prospectivo. Estes estudos são oriundos dos Estados Unidos (5 estudos), da Europa (3 estudos), Ásia (7 estudos), América Latina ( 1 estudo) e Oceania (1 estudo). O número de pacientes variou de 22 a 484, totalizando 2.223 pacientes estudados. Muitos desses pacientes foram submetidos à cirurgia cardíaca (5 estudos) $(26,27,32-34)$ ou também encontravam-se internados em UTI (4 estudos) $(29,30,35,36)$. Outras condições associadas ao desenvolvimento de LRA incluíram nefropatia induzida por contraste (NIC) (37), envenenamento pelo agrotóxico glifosato (38), sepse (39), malária (40), nefropatia obstrutiva (41), dentre outras $(31,42,43)$.

Em relação às metodologias mais comuns empregadas na determinação do biomarcador em amostras de urina, destaca-se o ensaio de imunoabsorção ligada à enzima (ELISA, do inglês Enzyme-Linked Immunosorbent Assay) que foi a metodologia mais empregada pelos estudos para a mensuração das concentrações de KIM-1 em amostras de urina (Tabela 1).

Tabela 1-Caracterísitcas gerais dos artigos selecionados.

\begin{tabular}{|c|c|c|c|c|c|c|}
\hline $\begin{array}{c}\text { Estudos } \\
\text { selecionados }\end{array}$ & Local & $\begin{array}{c}\text { Desenho do } \\
\text { Estudo }\end{array}$ & $\begin{array}{c}\text { Condição Clínica } \\
\text { dos Pacientes }\end{array}$ & Definição de LRA & $\begin{array}{c}\text { LRA/Sem LRA } \\
\text { (n) }\end{array}$ & Ensaio \\
\hline Akdeniz et al., 2015 (37) & Turquia & Coorte prospectivo & NIC & KDIGO & $32 / 88$ & ELISA \\
\hline Bouchard et al., 2015 (35) & Estados Unidos & Observacional prospectivo & Pacientes UTI & AKIN & $56 / 24$ & NR \\
\hline de Geus et al., 2013 (29) & Holanda & Coorte prospectivo & Pacientes UTI & AKIN & $49 / 432$ & ELISA \\
\hline Han et al., 2009 (27) & Estados Unidos & NR & Cinurgia cardiaca & AKIN & $36 / 54$ & ELISA \\
\hline Koyner et al., 2010 (32) & Estados Unidos & Coorte prospectivo & Cirurgia cardiaca & AKIN & $46 / 76$ & NR \\
\hline Liangos et al., 2009 (26) & Estados Unidos & Coorte prospectivo & Cirurgia cardiaca & AKIN & $13 / 90$ & ELISA \\
\hline Metzger et al., 2016 (33) & Alemanha & Coorte prospectivo & Cirurgia cardiaca & AKIN & $59 / 51$ & ELISA \\
\hline Mohamed et al., 2016 (38) & Sri Lanka & Coorte prospectivo & Envenenamento* & AKIN & $46 / 44$ & ELISA \\
\hline Morales-Buenrostro et al., 2014 (30) & México & Coorte prospectivo & Pacientes UTI & AKIN & $17 / 20$ & ELISA \\
\hline Ralib et al., 2012 (36) & Nova Zelândia & Coorte & Pacientes UTI & AKIN & $218 / 266$ & ELISA \\
\hline Ren et al., 2015 (42) & China & Coorte prospectivo & Queimados & KDIGO & $47 / 11$ & ELISA \\
\hline Sabbisetti et al., 2014 (34) & Estados Unidos & Coorte prospectivo & Cinurgia cardiaca & KDIGO & $28 / 16$ & Microbead based assay \\
\hline Tekce et al., 2014 (43) & Turquia & Coorte prospectivo & Tto. Cisplatina & AKIN & $8 / 14$ & ELISA \\
\hline Tu Y et al., 2014 (39) & China & NR & Sepse & AKIN & $49 / 101$ & ELISA \\
\hline Van Wolfswinkel et al., 2016 (40) & Holanda & Coorte & Malária & KDIGO & $6 / 33$ & ELISA \\
\hline Xue et al., 2014 (41) & China & Coorte prospectivo & Nefropatia obstrutiva & AKIN & $54 / 36$ & ELISA \\
\hline Yang et al., 2016 (31) & Taiwan & Observacional prospectivo & Falência cardíaca & KDIGO & $49 / 54$ & ELISA \\
\hline
\end{tabular}

* Envenenamento com o herbicida glifosato; ** Aumento significativo $(\mathrm{p}<0,05)$ de KIM-1 na urina dos apcientes que desenvolveram LRA em comparação aos pacientes sem LRA. KIM-1u: KIM-1 urinária; NIC: nefropatia induzida por contraste; NR: não relatado; Tto: tratamento; UTI: unidade de terapia intensiva.

\subsection{KIM-1 no diagnóstico precoce de LRA}

Na tabela 2 está representada a performance clínica da KIM-1 urinária no diagnóstico precoce de LRA. De maneira geral, observou-se nos estudos aumentos significativos nos níveis de KIM-1 na urina dos pacientes que desenvolveram LRA, comparado com os pacientes sem a lesão renal. $\mathrm{O}$ aumento do biomarcador ocorreu principalmente entre a admissão do paciente no hospital e em até 48 horas após a internação ou procedimentos aos quais os pacientes foram submetidos, dependendo do caso apresentado nos estudos. Os valores da AUC-ROC variaram de 0,57 - 1,0, e, os valores de sensibilidade e especificidade, que caracterizam o desempenho da KIM-1, foram de $35,71 \%$ a $100 \%$ e de $58 \%$ a $100 \%$, respectivamente (Tabela 2 ).

Nos pacientes submetidos a cirurgias cardíacas, o aumento nas concentrações de KIM-1 na urina foi observado imediatamente após a cirurgia (27) ou até 12 h após o procedimento (33). Esse aumento foi constatado antes da elevação da creatinina sérica (26). Neste mesmo grupo de pacientes, a determinação de KIM-1 na urina apresentou uma performance clínica moderada com valores de AUC-ROC de 0,57 - 0,78 e 
valores de sensibilidade e especificidade de $35,7 \%$ a $92 \%$ e $58 \%$ a $90 \%$, respectivamente $(26,27,32-34)$. No estudo de Liangos e colaboradores, KIM-1 urinária apresentou performance clínica (AUC-ROC: 0,78) superior no diagnóstico de LRA em pacientes submetido à cirurgia cardíaca, em comparação a outros biomarcadores como NGAL, IL-18, N-Acetil- $\beta$-d-glucosaminidase, cistatina $\mathrm{C}$ e $\alpha-1$ microglobulina (26). Além disso, em pacientes internados em UTI foi constatado um aumento significativo nas concentrações urinários de KIM-1 já na admissão (29, 30, 35, 36). A determinação de KIM-1 neste grupo de pacientes mostrou uma excelente capacidade diagnóstica do biomarcador, com uma AUC-ROC de 1,0 e valores de sensibilidade e especificidade de 100\% (30). KIM-1 também apresentou uma boa performance clínica (AUC-ROC: 0,9) na detecção de LRA em pacientes com nefropatia obstrutiva (41). De particular importânica, neste estudo, os autores também relataram o papel da KIM-1 na avaliação do prognóstico dos pacientes com LRA. Foi constatado que nos pacientes com nefropatia obstrutiva, que desenvolveram LRA, a concentração da KIM-1 na urina foi diretamente relacionada com a recuperação da função renal na maioria dos casos, ou seja, a concentração urinária da KIM-1 foi menor nos pacientes com LRA com subsequente recuperação da função renal em comparação aos pacientes que não tiveram a recuperação (41), indicando que a determinação da KIM-1 na urina, além de demonstrar potencial diagnóstico, é também capaz de prever potenciais desfechos clínicos dos pacientes.

Tabela2 - Performance clínica da KIM-1 no diagnóstico precoce de LRA.

\begin{tabular}{|c|c|c|c|c|c|c|c|}
\hline $\begin{array}{c}\text { Estudos } \\
\text { selecionados }\end{array}$ & $\begin{array}{c}\text { Condição Clínica } \\
\text { dos Pacientes }\end{array}$ & $\begin{array}{l}\text { Aumento significativo } \\
\text { da KIM-1u }(\operatorname{sim} / \mathrm{Não})^{* *}\end{array}$ & $\begin{array}{c}\text { Intervalo de Tempo } \\
\text { para o aumento da KIM-1u }\end{array}$ & Ponto de Corte & AUC-ROC & $\begin{array}{c}\text { Sensibilidade } \\
\% \\
\end{array}$ & $\begin{array}{c}\text { Especificidade } \\
\% \\
\end{array}$ \\
\hline Akdeniz et al., 2015 (37) & NIC & $\operatorname{sim}$ & $6 \mathrm{~h}$ após administração & NR & 0,797 & 75 & 75 \\
\hline Bouchard et al., 2015 (35) & Pacientes UTI & Não & NR & NR & 0,67 & NR & NR \\
\hline de Geus et al., 2013 (29) & Pacientes UTI & Sim & Admissão & NR & 0,73 & NR & NR \\
\hline Han et al., 2009 (27) & Cinurgia cardíaca & Sim & Imediatamente; 3h após cirurgia & $>1,2 \mathrm{ng} / \mathrm{mg} \mathrm{Cr}$ & 0,$68 ; 0,65$ & 51,$43 ; 35,71$ & 77,$78 ; 90,2$ \\
\hline Koyner et al., 2010 (32) & Cirurgia cardiaca & $\operatorname{sim}$ & NR & NR & 0,67 & NR & NR \\
\hline Liangos et al., 2009 (26) & Cirurgia cardiaca & Sim & 2h após cirurgia & $0,42 \mathrm{ng} / \mathrm{mg} \mathrm{Cr}$ & 0,78 & 92 & 58 \\
\hline Metzger et al., 2016 (33) & Cirurgia cardiaca & Sim & $12 \mathrm{~h}$ após cirurgia & $2,1 \mathrm{ng} / \mathrm{mL}$ & 0,57 & 60 & 67 \\
\hline Mohamed et al., 2016 (38) & Envenenamento* & Sim & $8 \mathrm{~h} ; 16 \mathrm{~h}$ & $\mathrm{NR}$ & 0,$73 ; 0,78$ & NR & NR \\
\hline Morales-Buenrostro et al., 2014 (30) & Pacientes UTI & $\operatorname{Sim}$ & 48h; $24 \mathrm{~h}$ antes da LRA & $16 \mathrm{ng} / \mathrm{mL} ; 26 \mathrm{ng} / \mathrm{mL}$ & 0,$91 ; 1,0$ & $83 ; 100$ & $95 ; 100$ \\
\hline Ralib et al., 2012 (36) & Pacientes UTI & Sim & Admissão & $\mathrm{NR}$ & 0,68 & NR & $\mathrm{NR}$ \\
\hline Ren et al., 2015 (42) & Queimados & $\operatorname{sim}$ & Admissão & $5,33 \mathrm{ng} / \mathrm{mL}$ & 0,846 & 72,7 & 86,9 \\
\hline Sabbisetti et al., 2014 (34) & Cirurgia cardiaca & Sim & $48 \mathrm{~h}$ & $\mathrm{NR}$ & 0,89 & NR & NR \\
\hline Tekce et al., 2014 (43) & Tto. Cisplatina & Sim & $24 \mathrm{~h} ; 48 \mathrm{~h}$ & $1412 \mathrm{pg} / \mathrm{mL}$ & 0,94 & 87,5 & 93,3 \\
\hline Tu Y et al., 2014 (39) & Sepse & Sim & $6 \mathrm{~h} ; 24 \mathrm{~h}$ & NR & 0,$86 ; 0,91$ & $94 ; 91$ & $61 ; 78$ \\
\hline Van Wolfswinkel et al., 2016 (40) & Malária & Sim & Admissão & $\geq 1,83 \mathrm{ng} / \mathrm{mL}$ & 0,87 & 100 & 72 \\
\hline Xue et al., 2014 (41) & Nefropatia obstrutiva & Sim & $72 \mathrm{~h}$ & $338,26 \mathrm{pg} / \mathrm{mg} \mathrm{Cr}$ & 0,9 & 90 & 75 \\
\hline Yang et al., $2016(31)$ & Falência cardiaca & Sim & Admissão & $1,62 \mathrm{ng} / \mathrm{mL}$ & 0,75 & 80 & 44 \\
\hline
\end{tabular}

* Envenenamento com o herbicida glifosato; ** Aumento significativo $(\mathrm{p}<0,05)$ de KIM-1 na urina dos pacientes que desenvolveram LRA em comparação aos pacientes sen LRA. Cre: creatinina; KIM-lu: KIM-1 urinária; NIC: nefropatia induzida por contraste; NR: não relatado; Tto: tratamento; UTI: unidade de terapia intensiva.

Akdeniz e colaboradores investigaram o papel da KIM-1 como marcador precoce de LRA em pacientes que receberam administração de contraste radiográfico. Neste estudo, a concentração urinária de KIM-1 aumentou significativamente 6 horas após administração de constraste, enquanto que os níveis de creatinina sérica aumentaram apenas 48 horas após a administração (37). Neste mesmo estudo, KIM-1 também apresentou boa acurácia com uma AUC-ROC de 0,79, indicandoque em pacientes com nefropatia induzida por contraste (NIC), a deteminação de KIM-1 na urina mostrou superioridade diagnóstica em relação a outros marcadores como a creatinina sérica (37). Em outro estudo, Tekce e colaboradores investigaram o papel de KIM-1 urinária no diagnóstico precoce de LRA induzida pela administração de cisplatina em 22 pacientes com tumores sólidos. A análise da curva de características operacionais do receptor revelou que a concentração urinária de KIM-1, 24 horas após administração de cisplatina, foi capaz de prever o desenvolvimento de LRA com uma sensibilidade 87,5\% e especificidade de 93,3\% (AUC-ROC: 0,94) (43).

Além disso, conforme mostrado na tabela 2, KIM-1 urinária apresentou uma boa performance no diagnóstico de LRA em pacientes com sépse (39). Neste estudo, os 
níveis urinários de KIM-1 aumentaram significativamente 6 horas após a hospitalização, com pico em 24 horas e permaneceram elevados até 48 horas após a internação (39). De maneira interessante, no mesmo estudo, foi observado que a elevação persistente da KIM-1 entre 24 e 48 horas após a admissão dos pacientes sépticos na UTI foi relacionada com um prognóstico desfavorável de mortalidade, visto que o aumento persistente do biomarcador foi encontrado majoritariamente em pacientes não sobreviventes (39). Desta forma, além de demonstrar um bom potencial no diagnóstico precoce de LRA, a determinação de KIM-1 na urina também se mostrou eficiente na previsão de potenciais prognósticos dos pacientes.

Por outro lado, KIM-1 urinária mostrou uma performance clínica moderada no diagnóstico de LRA em pacientes intoxicados com o herbicida glifosato em 8 (AUC-ROC: 0,73) ou 16 horas (AUC-ROC: 0,78) após intoxicação (38). Já em pacientes queimados, KIM-1 urinária se mostrou excelente como biomarcador de LRA (AUC-ROC: 0,846), apresentando alta sensibilidade e especificidade (42). Além disso, análises de correlação revelaram que os níveis urinários da KIM-1 foram correlacionados com a creatinina sérica, sugerindo que a determinação de KIM-1 na urina pode ser usada como marcador precoce de LRA em pacientes queimados (42). Além disso, outro estudo também relata uma boa performance clínica da KIM-1 no diagnóstico de LRA em pacientes com malária (AUC-ROC: 0,87) (40). De uma maneira geral, os estudos avaliados na presente revisão mostram que a KIM-1 urinária apresenta uma boa performance clínica para o diagnóstico precoce de LRA em diferentes condições clínicas. Além de demonstrar um bom potencial no diagnóstico precoce de LRA, a determinação de KIM-1 na urina também se mostrou eficiente na previsão de potenciais prognósticos dos pacientes.

Na tabela 2, para a maior dos estudos, está representado o valor do ponto de corte para a KIM-1. É possível observar variação nas unidades utlizadas na expressão das concentrações do biomarcador. A maioria dos estudos expressa os níveis da KIM-1 em $\mathrm{ng} / \mathrm{mL}$ ou em $\mathrm{pg} / \mathrm{mL}$; porém alguns estudos expressam os resultados corrigidos pela creatinina urinária ( $\mathrm{mg} / \mathrm{g}$ de creatinina). Atualmente não existe um consenso de qual seria a melhor forma de expressar os resultados para a KIM-1 e, ainda não está claro se os níveis da KIM-1 devem ser normalizados pela creatinina urinária. Vale ressaltar que a excreção urinária de creatinina pode variar em diversas situações (44-46), sendo que a correção da concentração da KIM-1 pela creatinina urinária pode não ser apropriada (47). Entretanto, mais estudos são necessários para esclarecer se a concentração urinária de KIM-1 deve ou não ser normalizada pela concentração de creatinina sérica.

\section{Considerações finais}

A necessidade de biomarcadores para estabelecer o diagnóstico precoce da LRA é de extrema relevância. A utilização de novos biomarcadores tem se mostrado essencial para reduzir a falta de precisão dos biomarcadores atualmente usados na prática clínica para o diagnóstico da LRA $(3,14)$. Nesta revisão crítica da literatura foram avaliados estudos primários sobre o uso clínico de KIM-1 urinária como potencial biomarcador na detecção precoce de LRA. Foi observado que, na grande maioria dos estudos avaliados, o aumento na concentração do biomarcador ocorreu de maneira significativa nos pacientes que desenvolveram LRA associada às mais diversas causas e, esse aumento ocorreu em intervalos de tempo muito curtos após intervenção clínica ou após lesão renal. Os estudos, de uma maneira geral, demonstraram uma boa performance clínica para KIM-1 no diagnóstico precoce de LRA.

Com objetivo de reduzir a morbidade e a mortalidade de pacientes com LRA, o diagnóstico precoce se faz necessário para que a efetiva intervenção seja feita o mais 
rápido possível. O biomarcador tradicional para diagnóstico de LRA, a creatinina sérica, não reflete de maneira sensível o dano renal, tendo em vista que seu aumento é tardio. Por conta disso, a determinação de KIM-1 na urina parece representar uma boa perspectiva clínica no diagnóstico de LRA. Os dados avaliados nesta revisão indicam que a KIM-1 urinária apresenta potencial como biomarcador precoce de LRA. Entretanto, nosso estudo apresenta algumas limitações. Uma limitação que pode ser destacada é o fato de terem sido utilizadas apenas duas bases de dados diferentes para a busca de artigos científicos (MEDLINE e LILACS), o que pode reduzir a identificação de estudos relevantes. Além disso, a seleção de artigos apenas em português e inglês pode ter gerado perdas de estudos elegíveis em outras línguas como em espanhol, por exemplo, destacando-se um possível viés de seleção. Outra limitação do nosso estudo é que não foram utilizadas ferramentas para avaliar a qualidade dos artigos que foram incluídos na revisão. A avaliação da qualidade metodológica dos estudos primários é importante para minimizar vieses. Finalmente, a falta de padronização na expressão dos resultados das dosagens de KIM-1 também representa uma limitação, a qual compromete a comparação, a análise e a síntese de dados entre os diferentes estudos.

Desta forma, estudos futuros são necessários para validar a performance clínica desse biomarcador e, além disso, como não existe um marcador urinário ideal para diagnóstico precoce de LRA, estudos que avaliam a associação de diferentes biomarcadores podem ser interessantes no intuito de melhorar a performance diagnóstica dos mesmos. Além disso, estudos futuros são necessários para determinar qual a melhor forma de expressar os resultados para KIM-1, se corrigida ou não pela creatinina urinária. Ainda, vale destacar que, até o presente momento, não existem valores de referência estabelecidos para este biomarcador e, apesar dos esforços para se estabelecer valores de referência para KIM-1 urinária, mais estudos são ainda necessários neste tema. Apesar destes detalhes a serem investigados, a determinação de KIM-1 urinária parece promissora na prática clínica como potencial biomarcador precoce de LRA.

\section{Referências}

1. Riella MC. Princípios de Nefrologia e Distúrbios Hidroeletrolíticos. 5 ed. Rio de Janeiro: Guanabara Koogan; 2014.

2. Khwaja A. KDIGO clinical practice guidelines for acute kidney injury. Nephron Clin Pract 2012; 120, c179-c184.

3. Hsu RK, McCulloch CE, Dudley RA, Lo LJ, Hsu CY. Temporal changes in incidence of dialysisrequiring AKI. Journal of the American Society of Nephrology: JASN 2013; 24 (1): 37-42.

4. Beker BM, Corleto MG, Fieiras C, Musso CG. Novel acute kidney injury biomarkers: their characteristics, utility and concerns. IntUrolNephrol 2018; 50(4):705-713.

5. Ali T, Khan I, Simpson W, Prescott G, Townend J, Smith W, et al. Incidence and Outcomes in Acute Kidney Injury: A Comprehensive Population-Based Study. J Am Soc Nephrol 2007; 18 (4): 1292-8.

6. Bagshaw SM, George C, Bellomo R. Changes in the incidence and outcome for early acute kidney injury in a cohort of Australian intensive care units. Critical Care 2007;11(3): 1-9.

7. Rewa O, Bagshaw SM. Acute kidney injury: epidemiology, outcomes and economics. Nat Rev Nephrol 2014; 10 (4): 193-207.

8. Leung KCW, Tonelli M, James MT. Chronic kidney disease following acute kidney injury: risk and outcomes. Nat Rev Nephrol 2012; 9 (2): 77-85.

9. Waikar SS, Betensky RA, Bonventre JV. Creatinine as the gold standard for kidney injury biomarker studies? Nephrol Dial Transplant 2009; 24: 3263-65.

10. Coca SG, Peixoto AJ, Garg AX, Krumholz HM, Parikh CR. The prognostic importance of a small acute decrement in kidney function in hospitalized patients: a systematic review and meta-analysis. Am J Kidney Dis 2007; 50 (5): 712-20. 
11. Cruz DN, Goh CY, Palazzuoli A, Slavin L, Calabro A, Ronco C, et al. Laboratory parameters of cardiac and kidney dysfunction in cardio-renal syndromes. Heart Fail Rev 2011;16 (6): 545-51.

12. Endre ZH, Pickering JW, Walker RJ. Clearance and beyond: the complementary roles of GFR measurement and injury biomarkers in acute kidney injury (AKI). Am J Physiol Renal Physiol 2011; 30: F697-F707.

13. McCullough PA, Haapio M, Mankad S, Zamperetti N, Massie B, Bellomo R, et al. Prevention of cardio-renal syndromes: workgroup statements from the 7th ADQI Consensus Conference. Nephrol Dial Transplant 2010; 25 (6): 1777-84.

14. Rosner MH. Urinary biomarkers for the detection of renal injury. Adv Clin Chem 2009; 49: 73-97.

15. Kashani K, Cheungpasitporn W, Ronco C. Biomarkers of acute kidney injury: the pathway from discovery to clinical adoption. ClinChem Lab Med 2017; 55 (8): 1074-89.

16. Schrezenmeier EV, Barasch J, Budde K, Westhoff T, Schmidt-Ott KM. Biomarkers in acute kidney injury - pathophysiological basis and clinical performance. ActaPhysiol 2017; 219: 556-74.

17. Sharma RK. Biomarkers of acute kidney injury - ScienceDirect. Clinical Queries: Nephrology 2012;1 (1): 13-17.

18. Coca SG, Yusuf B, Shlipak MG, Garg AX, Parikh CR. Long-term risk of mortality and other adverse outcomes after acute kidney injury: a systematic review and meta-analysis. Am J Kidney Dis 2009; 53 (6): 961-73.

19. Xie Y, Wang Q, Wang C, Qi C, Ni Z, Mou S. High urinary excretion of kidney injury molecule-1 predicts adverse outcomes in acute kidney injury: a case control study.Crit Care 2016; 20: 1-12.

20. Han WK, Bailly V, Abichandani R, Thadhani R, Bonventre JV.Kidney Injury Molecule-1 (KIM-1): a novel biomarker for human renal proximal tubule injury. Kidney Int 2002; 62: 237-44.

21. Ichimura T, Brooks CR, Bonventre JV. Kim-1/Tim-1 and immune cells: shifting sands. Kidney Int 2012; 81:809-11.

22. Bailly V, Zhang Z, Meier W, Cate R, Sanicola M, Bonventre JV. Shedding of kidney injury molecule-1, a putative adhesion protein involved in renal regeneration. J BiolChem 2002; 277: 39739-21.

23. Bonventre JV. Kidney injury molecule-1 (KIM-1): a urinary biomarker and much more. Nephrol Dial Transplant 2009; 24: 3265-68.

24. Ichimura T, Asseldonk EJ, Humphreys BD, Gunaratnam L, Duffield JS, Bonventre JV. Kidney injury molecule- 1 is a phosphatidylserine receptor that confers a phagocytic phenotype on epithelial cells. J Clin Invest 2008; 118: 1657-68.

25. Ismail OZ, Zhang X, Wei J, Haig A, Denker BM, Suri RS, Sener A1, Gunaratnam L. Kidney injury molecule-1 protects against Galpha12 activation and tissue damage in renal ischemia-reperfusion injury. Am J Pathol 2015; 185: 1207-15.

26. Liangos O, Tighiouart H, Perianayagam MC, Kolyada A, Han WK, Wald R, et al. Comparative analysis of urinary biomarkers for early detection of acute kidney injury following cardiopulmonary bypass. Biomarkers 2009;14 (6): 423-31.

27. Han WK, Wagener G, Zhu Y, Wang S, Lee HT. Urinary biomarkers in the early detection of acute kidney injury after cardiac surgery. Clin J Am SocNephrol 2009; 4 (5): 873-82.

28. Liu S, Che M, Xue S, Xie B, Zhu M, Lu R, et al. Urinary L-FABP and its combination with urinary NGAL in early diagnosis of acute kidney injury after cardiac surgery in adult patients. Biomarkers 2013;18 (1): 95-101.

29. de Geus HR, Fortrie G, Betjes MG, van Schaik RH, Groeneveld AB. Time of injury affects urinary biomarker predictive values for acute kidney injury in critically ill, non-septic patients. BMC Nephrol 2013;14 (273): 1-7.

30. Morales-Buenrostro LE, Salas-Nolasco OI, Barrera-Chimal J, Casas-Aparicio G, Irizar-Santana S, Pérez-Villalva R, et al. Hsp72 Is a Novel Biomarker to Predict Acute Kidney Injury in Critically Ill Patients.PLoS One 2014; 9 (10): e109407.

31. Yang CH, Chang CH, Chen TH, Fan PC, Chang SW, Chen CC, et al. Combination of Urinary Biomarkers Improves Early Detection of Acute Kidney Injury in Patients With Heart Failure. Circ J 2016; 80 (4):1017-23.

32. Koyner JL, Vaidya VS, Bennett MR, Ma Q, Worcester E, Akhter SA, et al. Urinary biomarkers in the clinical prognosis and early detection of acute kidney injury. Clin J Am Soc Nephrol 2010; 5 (12): 2154-65. 
33. Metzger J, Mullen W, Husi H, Stalmach A, Herget-Rosenthal S, Groesdonk HV, et al. Acute kidney injury prediction in cardiac surgery patients by a urinary peptide pattern: a case-control validation study. Crit Care 2016; 20 (1):157.

34. Sabbisetti VS, Waikar SS, Antoine DJ, Smiles A, Wang C, Ravisankar A, et al. Blood kidney injury molecule-1 is a biomarker of acute and chronic kidney injury and predicts progression to ESRD in type I diabetes. J Am Soc Nephrol 2014; 25 (10): 2177-86.

35. Bouchard J, Malhotra R, Shah S, Kao YT, Vaida F, Gupta A, et al. Levels of protein C and soluble thrombomodulin in critically ill patients with acute kidney injury: a multicenter prospective observational study. PLoS One 2015; 10 (3): e0120770.

36. Ralib AM, Pickering JW, Shaw GM, Devarajan P, Edelstein CL, Bonventre JV, et al. Test characteristics of urinary biomarkers depend on quantitation method in acute kidney injury. J Am Soc Nephrol 2012; 23 (2): 322-33.

37. Akdeniz D, Celik HT, Kazanci F, Yilmaz H, Yalcin S, Bilgic MA, et al. Is Kidney Injury Molecule 1 a Valuable Tool for the Early Diagnosis of Contrast-Induced Nephropathy? J Investig Med 2015; 63 (8): 930-4.

38. Mohamed F, Endre ZH, Pickering JW, Jayamanne S, Palangasinghe C, Shahmy S, et al. Mechanismspecific injury biomarkers predict nephrotoxicity early following glyphosate surfactant herbicide (GPSH) poisoning. Toxicol Lett 2016; 258: 1-10.

39. Tu Y, Wang H, Sun R, Ni Y, Ma L, Xv F, et al. Urinary netrin-1 and KIM-1 as early biomarkers for septic acute kidney injury. Ren Fail 2014; 36 (10): 1559-63.

40. Van Wolfswinkel ME, Koopmans LC, Hesselink DA, Hoorn EJ, Koelewijn R, van Hellemond JJ, et al. Neutrophil gelatinase-associated lipocalin (NGAL) predicts the occurrence of malaria-induced acute kidney injury. Malar J 2016; 15: 464.

41. Xue W, Xie Y, Wang Q, Xu W, Mou S, Ni Z. Diagnostic performance of urinary kidney injury molecule-1 and neutrophil gelatinase-associated lipocalin for acute kidney injury in an obstructive nephropathy patient. Nephrology (Carlton) 2014; 19 (4):186-94.

42. Ren H, Zhou X, Dai D, Liu X, Wang L, Zhou Y, et al. Assessment of urinary kidney injury molecule1 and interleukin-18 in the early post-burn period to predict acute kidney injury for various degrees of burn injury.BMC Nephrol 2015; 16: 142.

43. Tekce BK, Uyeturk U, Tekce H, Aktas G, Akkaya A. Does the kidney injury molecule-1 predict cisplatin-induced kidney injury in early stage? Ann Clin Biochem 2014; 52 (1): 88-94.

44. Verhave JC, Fesler P, Ribstein J, du Cailar G, Mimran A. Estimation of renal function in subjects with normal serum creatinine levels: influence of age and body mass index. Am J Kidney Dis 2005; 46: 233-41.

45. Beddhu S, Samore MH, Roberts MS, Stoddard GJ, Pappas LM, Cheung AK. Creatinine production, nutrition, and glomerular filtration rate estimation. J Am Soc Nephrol 2003; 14:1000-5.

46. Kaysen GA, Chertow GM, Adhikarla R, Young B, Ronco C, Levin NW. Inflammation and dietary protein intake exert competing effects on serum albumin and creatinine in hemodialysis patients. Kidney Int 2001; 60: 333-40.

47. Conti M, Moutereau S, Esmilaire L, Desbene C, Lallali K, Devanlay M, Durrbach A, Manivet P, Eschwege P, Loric S. Should kidney tubular markers be adjusted for urine creatinine? The example of urinary cystatin C. Clin Chem Lab Med 2009; 47(12):1553-56. 